European

Thyroid Journal
Eur Thyroid J 2018;7:289-293

DOI: $10.1159 / 000490892$
Received: April 20, 2018

Accepted after revision: June 13, 2018

Published online: July 20, 2018

\title{
Study of Deiodinase Type 2 Polymorphisms in Graves' Disease and Ophthalmopathy in a Swedish Population
}

\author{
Bushra Shahida ${ }^{a}$ Tereza Planck ${ }^{a, b}$ Peter Åsman ${ }^{c, d}$ Mikael Lantz ${ }^{a, b}$ \\ ${ }^{a}$ Department of Clinical Sciences, Diabetes and Endocrinology, Lund University, Malmö, Sweden; \\ ${ }^{b}$ Department of Diabetes and Endocrinology, Skåne University Hospital, Malmö, Sweden; \\ 'Department of Clinical Sciences, Ophthalmology, Lund University, Malmö, Sweden; \\ ${ }^{\mathrm{d}}$ Department of Ophthalmology, Skåne University Hospital, Malmö, Sweden
}

\section{Keywords}

Deiodinase type 2 - Graves' disease · Graves'

ophthalmopathy · Single nucleotide polymorphism .

Thr92Ala · rs225014 • rs225011 · rs12885300 · Free T3 .

Thyroid-stimulating hormone receptor antibodies

\begin{abstract}
Background: Deiodinase type 2 (DIO2) is an enzyme that catalyzes the production of the active form of thyroid hormone triiodothyronine (T3) from thyroxine (T4) and is important for maintaining intracellular T3 levels. Single nucleotide polymorphisms (SNPs) in DIO2 were associated with several diseases. The association of SNPs in $\mathrm{DIO} 2$ with Graves' disease (GD) was suggested in 2 Russian studies. Objectives: The aim of the study was to examine whether SNPs in DIO2 are associated with GD or Graves' ophthalmopathy (GO). Methods: Seven SNPs in the DIO2 gene - rs225014 (Thr92Ala), rs12885300, rs2267872, rs225011, rs224995, rs225015, and rs2267873 - were studied to assess their association with $\mathrm{GD}$ and $\mathrm{GO}$. In total, 712 patients with $\mathrm{GD}$ with $(n=311)$ or without $(n=399)$ ophthalmopathy and 1,183 sex-matched controls from Malmö, Sweden were analyzed. In GD patients with available data, the SNPs were examined
\end{abstract}

\section{KARGER}

E-Mail karger@karger.com

www.karger.com/etj for association with the levels of free T3, free T4, thyroidstimulating hormone receptor antibodies (TRAb), and thyroid-peroxidase antibodies (TPOAb). Results: Rs225011 was nominally associated with GD (OR 1.18, Cl 1.01-1.37, $p=$ 0.036). None of the SNPs were associated with GO. In GD patients, none of the SNPs were associated with the free-T4 (fT4), TRAb, or TPOAb levels. A weak, nonsignificant association was observed between free-T3 (fT3) levels and rs 225014 and rs 12885300 , separately. Conclusions: Rs 225011 in DIO2 was weakly associated with GD. The mechanism behind this association requires further study. None of the investigated common SNPs in DIO2 was significantly associated with GO, fT3, fT4, TRAb, or TPOAb in GD patients.

\footnotetext{
(C) 2018 European Thyroid Association Published by S. Karger AG, Basel
}

\section{Introduction}

Deiodinase type 2 (DIO2) is an enzyme that catalyzes the production of the active form of thyroid hormone triiodothyronine (T3) from thyroxine (T4) and is important for maintaining intracellular T3 levels [1]. DIO2 is also responsible for the production of a fraction of serum $\mathrm{T} 3$ (c) 2018 European Thyroid Association.

Published by S. Karger AG, Basel
Tereza Planck, MD, $\mathrm{PhD}$

Department of Endocrinology, Skåne University Hospital

Jan Waldenströms gata 24

SE-205 02 Malmö (Sweden)

E-Mail tereza.planck@med.lu.se 
in euthyroid and hypothyroid individuals [2]. DIO2 is expressed in the pituitary, brain, thyroid, myocardium, skeletal muscle [3, 4], and adipose tissue [5]. We demonstrated the expression of DIO2 in intraorbital adipose tissue in euthyroid individuals and in intraorbital adipose tissue in patients with active and chronic Graves' ophthalmopathy (GO) [6]. In this study, DIO2 was downregulated in chronic but not active GO compared with controls. We speculated that a consequence of the decreased level of DIO2 in intraorbital tissues may be the limited local bioavailability of T3, thus promoting GO. In the present study, we hypothesize that single nucleotide polymorphisms (SNPs) in DIO2 could play a role in individual susceptibility to develop local hypothyroidism and GO.

The SNP Thr92Ala (rs225014) in DIO2 was linked to and associated with Graves' disease (GD) in 2 Russian studies [7, 8]. The Ala92 allele of the Thr92Ala SNP caused a delayed secretion of T3 following a TRH-mediated acute rise in $\mathrm{TSH}$, which is consistent with a reduced intrathyroidal conversion of T4 into T3 [9]. In contrast, the $-258 \mathrm{G} / \mathrm{x}$ variant of rs 12885300 was associated with a reduced rate of acute TSH-stimulated free T4 secretion with normal T3 release from the thyroid [10]. These findings suggest that common polymorphisms in $\mathrm{DIO} 2$ can subtly affect the circulating levels of thyroid hormone and might modulate the thyroid hormone homeostasis, which is further supported by the association of the Thr92Ala SNP with psychological well-being and response to T3 or T4 treatment [11].

SNPs in DIO2 have been associated with obesity, insulin resistance, and type 2 diabetes [12-16]; however, some studies could not confirm such associations [17, 18]. Thr92Ala SNP has also been associated with decreased bone mass, increased bone turnover [19], and increased risk for osteoarthritis [20] and hypertension [21]. Most of these associations were independent of serum thyroid hormone levels, and this underscores the importance of peripheral regulation of thyroid hormone production.

The aim of the present study was to examine whether SNPs in DIO2 are associated with GD and/or GO in a Swedish population.

\section{Material and Methods}

\section{Study Subjects}

The study consisted of 712 patients (127 males and 585 females) with GD with $(n=311)$ or without $(n=399)$ ophthalmopathy and 1,183 controls ( 211 males and 972 females) from Malmö in southern Sweden (Table 1). A total of 521 patients (73\%) were selected
Table 1. Characteristics of the study population

\begin{tabular}{|c|c|c|c|c|}
\hline & Cases & $\%$ & Controls & $\%$ \\
\hline Number & 712 & & 1,183 & \\
\hline Age at inclusion, years ${ }^{\mathrm{a}}$ & $49+14$ & & $57+6$ & \\
\hline \multicolumn{5}{|l|}{ Gender } \\
\hline Male & 127 & 17.8 & 211 & 17.8 \\
\hline Female & 585 & 82.2 & 972 & 82.2 \\
\hline \multicolumn{5}{|l|}{ Ethnicity } \\
\hline Swedish & 531 & 74.6 & 833 & 70.4 \\
\hline European & 117 & 16.4 & 177 & 15.0 \\
\hline Other & 61 & 8.6 & 83 & 7.0 \\
\hline Missing & 3 & 0.4 & 90 & 7.6 \\
\hline \multicolumn{5}{|l|}{ Smoking } \\
\hline Yes & 288 & 40.4 & 343 & 29.0 \\
\hline No & 395 & 55.5 & 801 & 67.7 \\
\hline Missing & 29 & 4.1 & 39 & 3.3 \\
\hline \multicolumn{5}{|l|}{ Ophthalmopathy } \\
\hline Yes & 311 & 43.7 & 0 & 0.0 \\
\hline No & 399 & 56.0 & 1,183 & 100.0 \\
\hline Missing & 2 & 0.3 & 0 & 0 \\
\hline
\end{tabular}

${ }^{a}$ Data for age are presented as the mean \pm SD.

from the registry GD2002 in which clinical data on patients with GD have been collected since 2002 by a single endocrinologist. In total, 119 patients were recruited from the studies MFM (Malmö Preventive Project) [22] and MKC (Malmö Diet and Cancer Study) [23] on the basis of an ICD-10 diagnosis of E05.0 (GD) and H06.2 (GO). These studies were performed in the 1990 s as a primary preventive project (MFM) and a study investigating the association of diet and risk of cancer (MKC). Seventy-two patients were recruited from the TT-96 study as described previously [24]. GD diagnosis was made by an endocrinologist based on clinical symptoms and signs and biochemical hyperthyroidism in combination with the presence of thyroid-stimulating hormone (TSH)-receptor antibodies (TRAb) and/or diffuse uptake by technetium scintigraphy. GO diagnosis was made by an endocrinologist and/or ophthalmologist based on the presence of clinical signs, as previously described [24]. Eyelid retraction alone was not classified as GO. The controls were recruited from the MFM and MKC databases. The case and control groups were matched for sex. Regarding ethnicity, the origin of the individuals was assigned as Swedish, born in a European country other than Sweden, or born outside of Europe.

Free-T4 (fT4; 12-22 pmol/L), free-T3 (fT3; 3.6-6.3 pmol/L), thyroid peroxidase antibodies (TPOAb; $<34 \mathrm{kIE} / \mathrm{L})$, and TRAb $(<1.2$ IE/L) levels at diagnosis of GD were measured with ElectroChemiLuminiscence Immunoassay on Cobas (Roche) at the Department of Clinical Chemistry, Skåne University Hospital, Sweden.

All patients provided written informed consent, and the local research Ethics Committee approved the study. For characteristics of the subjects, see Table 1.

SNP Selection and Genotyping

SNPs were selected using data from the Hap Map consortium [25]. Applying the tag SNP approach reduces the amount of SNPs that must be genotyped to cover the genetic variation in a region. 
Therefore, the region surrounding each of the selected genes, including an extra $10 \mathrm{~kb}$ upstream and downstream, was analyzed by Tagger in the Haplowiew program [26], providing tag SNPs. In total, 7 SNPs in the DIO2 gene, including rs225014, rs12885300, rs2267872, rs225011, rs224995, rs225015, and rs2267873, were selected for the analysis. The minor allele frequency for all SNPs was $>0.05$. DNA was extracted from whole blood using the MaxiPrep Kit (QIAGEN, Sweden), and SNPs were genotyped using the Sequenom platform (MALDI-TOF) and TaqMan SNP Genotyping using Quantstudio 7 Flex system. The genotyping was performed in our laboratory. SNPs included in the analysis exhibited a $>90 \%$ success rate.

\section{Power Calculations}

As the allele frequencies of the SNPs in our population were unknown prior to genotyping in this study, we based our power calculations on the rs225014 SNP where the allele frequencies were known from other studies $[13,15]$ and the Hap Map consortium using the Genetic Power Calculator [27]. Given a control/case ratio of 1.7 , minor allele frequency 0.40 and OR 1.3 , the number of cases required for $80 \%$ power and 0.05 significance is 710.

\section{Statistical Analysis}

Logistic regression using age, smoking and ethnicity as covariates was used to estimate SNP associations with GD and GO. In GD patients with available data, we examined possible associations of the DIO2 SNPs with the serum levels of fT3, fT4, TRAb, and anti-TPO upon diagnosis of GD. Linear regression adjusted for age was used to estimate associations with $\mathrm{fT} 3$ given that age was highly associated with fT3 levels in our material. Unadjusted associations were used for fT4, TRAb, and anti-TPO. The $p$ values are based on additive models for the genetic variants. Correction for multiple testing was performed using permutations (maxT permutation method in PLINK). All statistical calculations were performed using PLINK version 1.07 (http://pngu.mgh.harvard. edu/ purcell/plink/index.shtml) [28].

\section{Results}

\section{Association with GD}

Using logistic regression with age, smoking, and ethnicity as covariates, we found that rs225011 was associated with GD (OR 1.18, CI 1.01-1.37, $p=0.036)$. The remaining SNPs (i.e., rs225014, rs12885300, rs2267872, rs224995, rs225015, and rs2267873) were not associated with GD (Table 2). After correction for multiple testing, the association was no longer significant.

\section{Associations with GO, fT4, fT3, TRAb, and TPOAb}

None of the SNPs were associated with GO and serum levels of fT4, TRAb, or TPOAb. A weak, non-significant association for rs225014 (Thr92Ala) and rs12885300 with serum levels of fT3 was noted in GD patients with available data on fT3 at diagnosis $(n=377$; Table 3$)$.

DIO2 Polymorphisms in Graves' Disease and Ophthalmopathy
Table 2. Association of 7 DIO2 SNPs with Graves' disease

\begin{tabular}{lllll}
\hline SNP & Allele & OR & $95 \%$ CI & $p$ value \\
\hline rs225014 & C & 1.13 & $0.96-1.32$ & 0.15 \\
rs12885300 & T & 0.93 & $0.79-1.1$ & 0.39 \\
rs2267872 & A & 1.11 & $0.86-1.44$ & 0.42 \\
rs225011 & C & 1.18 & $1.01-1.37$ & 0.04 \\
rs224995 & C & 1.09 & $0.88-1.35$ & 0.41 \\
rs225015 & A & 1.10 & $0.93-1.29$ & 0.27 \\
rs2267873 & G & 0.98 & $0.76-1.25$ & 0.85 \\
\hline
\end{tabular}

Analysis was performed using logistic regression with age, smoking, and ethnicity as covariates.

Table 3. Association between DIO2 SNPs and serum fT3 (linear regression)

\begin{tabular}{llrll}
\hline SNP & Allele & Beta & \multicolumn{1}{l}{$95 \%$ CI } & $p$ value \\
\hline rs225014 & C & 1.40 & -0.13 to 2.94 & 0.07 \\
rs12885300 & T & -1.58 & -3.31 to 0.15 & 0.07 \\
rs2267872 & A & -0.29 & -2.40 to 1.81 & 0.78 \\
rs225011 & C & 1.16 & -0.32 to 2.65 & 0.12 \\
rs224995 & C & -0.74 & -2.72 to 1.25 & 0.46 \\
rs225015 & A & 1.21 & -0.31 to 2.74 & 0.12 \\
rs2267873 & G & -0.88 & -2.82 to 1.06 & 0.38 \\
\hline
\end{tabular}

Beta, regression coefficient.

\section{Discussion}

In this large Swedish case-control study of GD, we examined possible associations of common variants in the DIO2 gene and GD and GO, respectively. Our study is the first to examine associations of DIO2 SNPs and GD in a Scandinavian population and the first to study associations with GO. The only positive finding was an association between the rs225011 and GD, which, however, disappeared after correction for multiple testing. None of the SNPs were associated with GO. In GD patients, we did not observe any association between any of the SNPs and TRAb, TPOAb, or fT4 levels. An association was noted between 2 DIO2 SNPs, Thr92Ala (rs225014), and rs12885300, and circulating fT3 levels; however, the association was not statistically significant.

Our study reports similar conclusions as the findings of 2 earlier studies examining associations between SNPs in DIO2 and GD. In the Russian family study, SNPs located within the GD-1 susceptibility locus on chromosome 14q23-q32 were examined for predisposition to GD using the transmission disequilibrium test in 126 simplex 
Russian families affected with GD. Among the SNPs tested, a significant preferential transmission of the Ala allele of the Thr92Ala SNP from parents to affected children was identified, suggesting the association of the Thr92Ala SNP with GD [7]. In the more recent association study, the AA genotype of the Ala92Thr polymorphism of DIO2 gene was found to be protective regarding the development of GD, severity of disease, and remission rate in GD patients [8]. In our study, rs225011 but not Thr92Ala was weakly associated with GD. However, these 2 SNPs are in high linkage disequilibrium $\left(D^{\prime} \geq 0.9\right)$ and therefore are most likely inherited together [16]. The mechanism behind this association remains unknown as lack of association was noted between rs 225011 and TRAb, TPOAb, fT4, and fT3 levels in GD patients. Rs225011 was modestly associated with early-onset type 2 diabetes and hepatic glucose output in Pima Indians, the subjects with the risk allele exhibiting lower rates. The authors hypothesize that lower physiologically active plasma or intracellular T3 levels in the carriers of the risk allele could explain the reduced hepatic gluconeogenesis and association with diabetes [16]. GD is a multifactorial disease caused by interplay among endogenous, genetic, and environmental factors. Based on the weak OR of the associations in all studies, including ours, the SNPs in DIO2 probably contribute to the development and modulate the course of GD in the presence of TRAb rather than cause the disease. TRAb increases intrathyroidal DIO2 expression and activity, and increased intrathyroidal DIO2 activity may contribute significantly to the relative increase in thyroidal $\mathrm{T} 3$ production in GD patients [4]. Two Japanese studies aiming at characterizing the T3-predominant type of GD observed overexpression and increased thyroidal DIO2 activity as well as a high serum $\mathrm{fT} 3 / \mathrm{fT} 4$ ratio in patients with T3-predominant GD $[29,30]$. Thus, DIO2 gene polymorphisms could further modulate these responses. In our study, Thr92Ala and rs 12885300 exhibited weak, non-significant associations with circulating fT3 levels. It is still possible that a stronger association with $\mathrm{T} 3$ levels exists in the peripheral tissues, including the thyroid or orbital tissues.

The development of hypothyroidism in GD patients is a known risk factor for the development or worsening of GO. Therefore, we hypothesized that polymorphisms in the DIO2 gene could affect intraorbital levels of T3, thus influencing the risk of the development of GO. In the GD cohort, we did not observe any associations between the SNPs studied and the presence of GO. This mechanism does not seem to modulate the risk of $\mathrm{GO}$ in our population.

In conclusion, rs 225011 was weakly associated with GD in our study. The mechanism behind this association is currently not known. None of the investigated common SNPs in DIO2 were significantly associated with $\mathrm{GO}, \mathrm{fT} 4$, TRAb, or TPOAb in GD patients.

\section{Disclosure Statement}

The authors declare that there is no conflict of interest regarding the publication of this article.

\section{Funding Source}

This work was supported by grants from Svenska Läkaresällskapet and ALF Region Skåne.

\section{References}

1 Bianco AC, Kim BW: Deiodinases: implications of the local control of thyroid hormone action. J Clin Invest 2006;116:2571-2579.

2 Maia AL, Kim BW, Huang SA, Harney JW, Larsen PR: Type 2 iodothyronine deiodinase is the major source of plasma T3 in euthyroid humans. J Clin Invest 2005;115:2524-2533.

3 Croteau W, Davey JC, Galton VA, St Germain DL: Cloning of the mammalian type II iodothyronine deiodinase. A selenoprotein differentially expressed and regulated in human and rat brain and other tissues. J Clin Invest 1996;98:405-417.

4 Salvatore D, Tu H, Harney JW, Larsen PR: Type 2 iodothyronine deiodinase is highly expressed in human thyroid. J Clin Invest 1996; 98:962-968

5 Nomura E, Toyoda N, Harada A, Nishimura K, Ukita C, Morimoto S, Kosaki A, Iwasaka T,
Nishikawa M: Type 2 iodothyronine deiodinase is expressed in human preadipocytes. Thyroid 2011;21:305-310.

6 Planck T, Parikh H, Groop L, Hallengren B, Lantz M: Intraorbital deiodinase type 2 expression is downregulated in chronic phase of Graves' ophthalmopathy. Clin Endocrinol 2012;77:486-487.

7 Chistiakov DA, Savost'anov KV, Turakulov RI: Screening of SNPs at 18 positional candidate genes, located within the GD-1 locus on chromosome 14q23-q32, for susceptibility to Graves' disease: a TDT study. Mol Genet Metab 2004;83:264-270.

8 Alina B, Daria P, Olga F, Vladislav S, Anna $\mathrm{K}$, Elena G: Thr92Ala polymorphism of hD2 affects the development of Graves' disease, treatment efficiency, and rate of remission. Clin Dev Immunol 2012;2012:340542.
9 Butler PW, Smith SM, Linderman JD, Brychta RJ, Alberobello AT, Dubaz OM, Luzon JA, Skarulis MC, Cochran CS, Wesley RA, Pucino F, Celi FS: The Thr92Ala 5' type 2 deiodinase gene polymorphism is associated with a delayed triiodothyronine secretion in response to the thyrotropin-releasing hormone-stimulation test: a pharmacogenomic study.Thyroid 2010;20:14071412.

10 Peltsverger MY, Butler PW, Alberobello AT, Smith S, Guevara Y, Dubaz OM, Luzon JA, Linderman J, Celi FS: The -258A/G (SNP rs12885300) polymorphism of the human type 2 deiodinase gene is associated with a shift in the pattern of secretion of thyroid hormones following a TRH-induced acute rise in TSH. Eur J Endocrinol 2012;166:839845 . 
11 Panicker V, Saravanan P, Vaidya B, Evans J, Hattersley AT, Frayling TM, Dayan CM: Common variation in the $\mathrm{DIO} 2$ gene predicts baseline psychological well-being and response to combination thyroxine plus triiodothyronine therapy in hypothyroid patients. J Clin Endocrinol Metab 2009;94:1623-1629.

12 Mentuccia D, Proietti-Pannunzi L, Tanner K, Bacci V, Pollin TI, Poehlman ET, Shuldiner AR, Celi FS: Association between a novel variant of the human type 2 deiodinase gene Thr92Ala and insulin resistance: evidence of interaction with the Trp64Arg variant of the beta-3-adrenergic receptor. Diabetes 2002;51: 880-883.

13 Grarup N, Andersen MK, Andreasen CH, Albrechtsen A, Borch-Johnsen K, Jorgensen T, Auwerx J, Schmitz O, Hansen T, Pedersen O: Studies of the common DIO2 Thr92Ala polymorphism and metabolic phenotypes in 7342 Danish white subjects. J Clin Endocrinol Metab 2007;92:363-366.

14 Canani LH, Capp C, Dora JM, Meyer EL, Wagner MS, Harney JW, Larsen PR, Gross JL, Bianco AC, Maia AL: The type 2 deiodinase $\mathrm{A} / \mathrm{G}$ (Thr92Ala) polymorphism is associated with decreased enzyme velocity and increased insulin resistance in patients with type 2 diabetes mellitus. J Clin Endocrinol Metab 2005; 90:3472-3478.

15 Dora JM, Machado WE, Rheinheimer J, Crispim D, Maia AL: Association of the type 2 deiodinase Thr92Ala polymorphism with type 2 diabetes: case-control study and metaanalysis. Eur J Endocrinol 2010;163:427-434.

16 Nair S, Muller YL, Ortega E, Kobes S, Bogardus C, Baier LJ: Association analyses of variants in the DIO2 gene with early-onset type 2 diabetes mellitus in Pima Indians. Thyroid 2012;22:80-87.

17 Mentuccia D, Thomas MJ, Coppotelli G, Reinhart LJ, Mitchell BD, Shuldiner AR,
Celi FS: The Thr92Ala deiodinase type 2 (DIO2) variant is not associated with type 2 diabetes or indices of insulin resistance in the old order of Amish. Thyroid 2005;15:12231227.

18 Maia AL, Dupuis J, Manning A, Liu C, Meigs JB, Cupples LA, Larsen PR, Fox CS: The type 2 deiodinase (DIO2) A/G polymorphism is not associated with glycemic traits: the Framingham Heart Study. Thyroid 2007;17:199202.

19 Heemstra KA, Hoftijzer H, van der Deure WM, Peeters RP, Hamdy NA, Pereira A, Corssmit EP, Romijn JA, Visser TJ, Smit JW: The type 2 deiodinase Thr92Ala polymorphism is associated with increased bone turnover and decreased femoral neck bone mineral density. J Bone Miner Res 2010;25:13851391.

20 Meulenbelt I, Min JL, Bos S, Riyazi N, Houwing-Duistermaat JJ, van der Wijk HJ, Kroon HM, Nakajima M, Ikegawa S, Uitterlinden AG, van Meurs JB, van der Deure WM, Visser TJ, Seymour AB, Lakenberg N, van der Breggen R, Kremer D, van Duijn CM, Kloppenburg M, Loughlin J, Slagboom PE: Identification of DIO2 as a new susceptibility locus for symptomatic osteoarthritis. Hum Mol Genet 2008;17:1867-1875.

21 Gumieniak O, Perlstein TS, Williams JS, Hopkins PN, Brown NJ, Raby BA, Williams GH: Ala92 type 2 deiodinase allele increases risk for the development of hypertension. Hypertension 2007;49:461-466.

22 Nilsson PM, Nilsson JA, Berglund G: Population-attributable risk of coronary heart disease risk factors during long-term follow-up: the Malmo Preventive Project. I Intern Med 2006;260:134-141.

23 Manjer J, Carlsson S, Elmstahl S, Gullberg B, Janzon L, Lindstrom M, Mattisson I, Berglund G: The Malmo Diet and Cancer Study: representativity, cancer incidence and mortality in participants and non-participants. Eur J Cancer Prev 2001;10:489-499.

24 Traisk F, Tallstedt L, Abraham-Nordling M, Andersson T, Berg G, Calissendorff J, Hallengren B, Hedner P, Lantz M, Nystrom E, Ponjavic V, Taube A, Torring O, Wallin G, Asman P, Lundell G: Thyroid-associated ophthalmopathy after treatment for Graves' hyperthyroidism with antithyroid drugs or iodine-131. J Clin Endocrinol Metab 2009;94: 3700-3707.

25 International HapMap Consortium: The International HapMap Project. Nature 2003; 426:789-796.

26 Barrett JC, Fry B, Maller J, Daly MJ: Haploview: analysis and visualization of $\mathrm{LD}$ and haplotype maps. Bioinformatics 2005;21:263265.

27 Purcell S, Cherny SS, Sham PC: Genetic power calculator: design of linkage and association genetic mapping studies of complex traits. Bioinformatics 2003;19:149-150.

28 Purcell S, Neale B, Todd-Brown K, Thomas L, Ferreira MA, Bender D, Maller J, Sklar P, de Bakker PI, Daly MJ, Sham PC: PLINK: a tool set for whole-genome association and population-based linkage analyses. Am J Hum Genet 2007;81:559-575.

29 Ito M, Toyoda N, Nomura E, Takamura Y, Amino N, Iwasaka T, Takamatsu J, Miyauchi A, Nishikawa M: Type 1 and type 2 iodothyronine deiodinases in the thyroid gland of patients with 3,5,3' -triiodothyronine-predominant Graves' disease. Eur J Endocrinol 2011; 164:95-100.

30 Matsumoto C, Ito M, Yamada H, Yamakawa N, Yoshida H, Date A, Watanabe M, Hidaka Y, Iwatani Y, Miyauchi A, Takano T: Genes that characterize T3-predominant Graves' thyroid tissues. Eur J Endocrinol 2013;168:137144 